\title{
BMJ Open Burden of tinea capitis among children in Africa: protocol for a systematic review and meta-analysis of observational studies, 1990-2020
}

\author{
Felix Bongomin (D) , ${ }^{1}$ Ronald Olum (D) , ${ }^{2}$ Lauryn Nsenga (D) , ${ }^{3}$ \\ Joseph Baruch Baluku (D) 4,5
}

To cite: Bongomin F, Olum R, Nsenga $L$, et al. Burden of tinea capitis among children in Africa: protocol for a systematic review and meta-analysis of observational studies, 1990-2020. BMJ Open 2020;10:e041230. doi:10.1136/ bmjopen-2020-041230

- Prepublication history for this paper is available online. To view these files, please visit the journal online (http://dx.doi. org/10.1136/bmjopen-2020041230).

Received 03 June 2020 Revised 01 August 2020 Accepted 19 August 2020

Check for updates

(C) Author(s) (or their employer(s)) 2020. Re-use permitted under CC BY-NC. No commercial re-use. See rights and permissions. Published by BMJ.

${ }^{1}$ Department of Medical Microbiology and Immunology, Faculty of Medicine, Gulu University, Gulu, Uganda ${ }^{2}$ School of Medicine, Makerere University College of Health Sciences, Kampala, Uganda ${ }^{3}$ School of Medicine, Kabale University, Kabale, Uganda ${ }^{4}$ Division of Pulmonology, Mulago National Referral Hospital, Kampala, Uganda ${ }^{5}$ Department of Programs, Mildmay Uganda, Wakiso, Uganda

Correspondence to Dr Felix Bongomin; drbongomin@gmail.com

\section{ABSTRACT}

Introduction Tinea capitis is the most common form of dermatophytosis among children, contributing significantly to the global burden of skin and hair infections. However, an accurate account of its burden in Africa, where most cases are thought to occur, is lacking. We aim to systematically evaluate the burden, aetiology and epidemiological trend of tinea capitis among children over a 30-year period in Africa.

Methods and analysis A systematic review will be conducted using Embase, PubMed, African Journals Online, Web of Science and the Cochrane Library of Systematic Review. These resources will be used to identify studies published between 1990 and December 2020, which report the prevalence, aetiology and trend of tinea capitis among children younger than 18 years in Africa. Articles in English and French will be considered. Two independent reviewers will screen the articles for eligibility, and any discrepancies will be resolved by discussion and consensus between the authors. Methodological quality of all studies will be assessed and critically appraised. We will perform a metaregression to assess the impact of study characteristics on heterogeneity and also to correct the meta-analytical estimates for biases. A qualitative synthesis will be performed, and STATA V.16.0 software will be used to estimate the pooled prevalence and aetiology of tinea capitis. The Mann-Kendall trend test will be use to evaluate the trend in the prevalence of tinea capitis over the study period.

Ethics and dissemination Ethical approval from an institutional review board or research ethics committee is not required for this systematic review and meta-analysis. The results will be published in a peer-reviewed journal and presented in conferences.

\section{INTRODUCTION}

Tinea capitis (or scalp ringworm) is a cutaneous fungal infection predominantly occurring in children between 3 and 7 years of age, but can occur in those as young as 6 months to $10-12$ years. $^{12}$ It is typically characterised by scalp erythema, hair loss, scaling and crusting. The global burden of tinea capitis is unknown, but the majority of cases are
Strengths and limitations of this study

- This will be the first systematic review and metaanalysis evaluating the burden, aetiology and trend of tinea capitis among children over a 30 -year period in Africa.

- A systematic search strategy including the most prominent databases in health and African Journals Online, a database for African-based research publications.

- We will attempt to evaluate the impact of HIV infection on the prevalence and aetiology of tinea capitis among children in Africa.

- We anticipate that some of the studies may have diagnosed tinea capitis clinically.

- HIV status may be missing or unknown in many of the studies.

reported in prepubertal children or in children of sub-Saharan African descent living abroad. The disease is mainly observed among children of low socioeconomic status and high population densities, and among those with poor health and sanitation practices, typically seen in low-income countries. ${ }^{34}$ This infection continues to be a neglected entity of global and public health concern. ${ }^{5}$

Tinea capitis is caused by a keratindegrading group of moulds collectively known as dermatophytes whose distribution varies geographically, demographically and with socioeconomic status. ${ }^{6}$ Recent phylogenetic reclassification of dermatophytes classifies them under nine genera with over 50 species. However, there are only a few species belonging to the genera Epidermophyton, Microsporum, Trichophyton, Arthroderma and Nannizzia, and these are commonly encountered in clinical practice. ${ }^{6}$ Dermatophytes are encountered globally, but the causative agents of tinea capitis vary significantly from one region of the world to another. An 
understanding of the changing epidemiology and the distribution of the aetiological species is important in deciding appropriate therapy and preventive approaches. ${ }^{7}$

The pattern of disease transmission can be humanto-human (anthroponoses, by anthropophilic dermatophytes, such as Trichophyton schoenleinii) or animal-to-humans (zoonoses, by zoophilic dermatophytes, such as Microsporum canis) and, rarely, from soilto-human (by geophilic dermatophytes, such as Nannizzia gypsea $){ }^{6}$ A clear understanding of these complex transmission dynamics is key in the control and prevention of tinea capitis. Anthropophilic organisms cause endemic infections in a large number of children or outbreaks in congregate settings such as schools; meanwhile, the zoophilic and geophilic organisms are usually associated with a sporadic but more inflammatory disease. ${ }^{8}$

The diagnosis of these infections is mainly clinical, ${ }^{9}$ with patchy hair loss in a ringed pattern being almost always pathognomonic of ringworm. However, alopecia areata, seborrheic and atopic dermatitis, and bacterial folliculitis can mimic the diagnosis of tinea capitis. ${ }^{10}$ Therefore, microscopic demonstration of characteristic microconidia/macroconidia and evaluation of culture characteristics on a mycological agar are required for definitive confirmation of the aetiological agent. ${ }^{10}$ The gross anatomy of the lesion, geographical location of the patient, travel history and history of contact with animals are all important clues for diagnosing the cause of the disease. ${ }^{11}$ On dermoscopy, arthrospores may be observed outside of the hair shaft (ectothrix pattern), inside the hair shaft (endothrix), and in and outside of the hair shaft (endoectothrix), or a complete destruction (favus) of the hair shaft may occur. ${ }^{8}$ Species-level or subspecies-level identification using molecular techniques is widely used in the Western world; however, it is not routinely used in resource-limited settings. ${ }^{8}$

While an earlier study showed that dermatophytosis occurs in between $10 \%$ and $70 \%$ of children throughout Africa, with tinea capitis being the most common presentation, ${ }^{12}$ an accurate estimate of the true burden of tinea capitis in Africa remains unknown. An accurate quantification of the burden of this neglected tropical disease is required to inform clinical and public health intervention strategies.

\section{Objectives}

This study seeks to systematically evaluate the burden, aetiology and trend of tinea capitis among children in Africa over a 30-year period.

\section{METHODS AND ANALYSIS}

This systematic review and meta-analysis protocol has been developed according to recommendations by Meta-analyses of Observational Studies in Epidemiology (MOOSE) ${ }^{13}$ and the Preferred Reporting Items for Systematic Review and Meta-Analysis Protocols checklists ${ }^{14}$ provided in online supplemental material 1 and online supplemental material 2, respectively. This protocol has been registred with PROSPERO (submission ID number:189727)

\section{Patient and public involvement}

No patients or the public will be involved in this systematic review and meta-analysis.

\section{Search strategy}

With the help of a qualified medical librarian, we will systematically search all studies published from January 1990 to December 2020 from the following databases: Embase, PubMed, African Journals Online, Web of Science and the Cochrane Library of systematic review. Authors will be contacted for eligible studies where only abstracts are accessible.

The following MeSH search terms will be used: "tinea capitis," "dermatophytes," "dermatophytosis," "scalp infections," "scalp ringworm," "Trichophyton," "Microsporum," "Epidermophyton," "Nannizzia," "Arthroderma," "burden," "prevalence," "incidence," AND "Africa," OR each of the individual 54 countries of Africa. The MeSH search terms shall also be translated into French and the same search strategy repeated.

We will also perform a manual literature search of all the references included in the articles that meet the selection criteria. The search will be limited to human studies and those written in English and French, the two most written languages in African publications, will be selected. A bilingual scientist will translate the French articles into English for review and data extraction by the authors.

\section{Study selection and inclusion criteria}

The studies found through databases that are duplicates will be removed using the Healthcare Databases Advanced Search (HDAS) programme. The records will initially be screened by title and abstract independently by two reviewers (FB and $\mathrm{RO}$ ) to exclude those not related to this study. The full texts of studies that pass the initial screening will be retrieved and assessed. Any disagreements will be resolved by discussion and consensus from all authors (FB, RO, JBB and LN).

The MOOSE criteria for searching and selecting observational studies will be used: the following inclusion criteria will be applied to identify eligible studies:

1. Observational studies published from January 1990 to December 2020 and in the English and French languages.

2. Designed as retrospective or prospective observational study.

3. Among children $(<18$ years old) in African countries.

4. Reporting the prevalence or incidence of tinea capitis. The following studies shall be excluded:

1. Case reports, case series with subjects less than 10 , opinion papers, qualitative research, letters to the editor, comments, conference proceedings, policy papers, reviews and meta-analyses, study protocols without baseline data and animal studies. 
2. Studies reporting other scalp infections.

\section{Data extraction and management}

A data extraction form will be prepared using Microsoft Excel V.2016 to capture information on study characteristics, participants and study outcomes (ie, prevalence and culture-proven aetiology). The form will be used by two independent reviewers (FB and RO) who will extract and code the data. Data extracted by the two reviewers will be verified and validated, and in case of any variation, it will be discussed and resolved by reviewers. Missing or ambiguous information will be obtained or clarified by contacting original authors of included studies where possible. The data will be stored in the authors' personal computers and online cloud as a secondary backup.

\section{Study outcomes}

Primary outcome

Prevalence of tinea capitis among children in Africa.

\section{Secondary outcome}

1. Culture proven aetiology, reported to at least genus level and trend in the prevalence of tinea capitis over the 30-year study period.

2. To evaluate the prevalence, aetiology and trend of tinea capitis among children with HIV infection.

\section{Quality assessment}

Two reviewers (LN and JBB), using the Critical Appraisal Skills Programme quality assessment tool, will independently perform assessment of the risk of bias in the selected studies. Different components including study design, selection bias, measurement of outcomes and the study findings will be assessed for each study. Each of the individual components and the overall study rating will be graded into categories of risk of bias as low, moderate and high. The overall grade will be obtained as an average of the individual components. Any discrepancies will be resolved by discussion and a third reviewer when necessary.

\section{Data synthesis and analysis}

We will use Microsoft Excel V.2016 and STATA V.16.0 software for analysis. A random-effect model meta-analysis will be performed using metaprop command for analysis of proportions in STATA and presented as prevalence, 95\% CIs and weights. A forest plot will be used to present the results of the meta-analysis. Sensitivity analysis will also be performed. A systematic descriptive synthesis will be performed appropriately to complete the meta-analysis. Heterogeneity of studies will be assessed using $Q$ statistics; $I^{2}$ and $\mathrm{p}$ value will be used to report heterogeneity between studies. We will perform a metaregression to assess the impact of study characteristics on heterogeneity and also to correct the meta-analytical estimates for biases. A p value of $<0.05$ will be considered statistically significant. The Mann-Kendall trend test will be used to evaluate the trend in the prevalence of tinea capitis over the study period.

\section{ETHICS AND DISSEMINATION}

The final results of this study will be published in an open-access peer-reviewed journal. This systematic review and meta-analysis will report the burden of tinea capitis in Africa and guide public health interventions. This review will be updated every 10 years as new publications become available.

\section{Twitter Felix Bongomin @dr_bongomin}

Contributors FB conceived the study and drafted the manuscript, and is the guarantor of this systematic review protocol. RO, LN and JBB drafted and revised the manuscript. All authors approved the final version of the manuscript.

Funding The authors have not declared a specific grant for this research from any funding agency in the public, commercial or not-for-profit sectors.

Competing interests None declared.

Patient and public involvement Patients and/or the public were not involved in the design, conduct, reporting or dissemination plans of this research.

Patient consent for publication Not required.

Provenance and peer review Not commissioned; externally peer reviewed.

Open access This is an open access article distributed in accordance with the Creative Commons Attribution Non Commercial (CC BY-NC 4.0) license, which permits others to distribute, remix, adapt, build upon this work non-commercially, and license their derivative works on different terms, provided the original work is properly cited, appropriate credit is given, any changes made indicated, and the use is non-commercial. See: http://creativecommons.org/licenses/by-nc/4.0/.

\section{ORCID iDs}

Felix Bongomin http://orcid.org/0000-0003-4515-8517

Ronald Olum http://orcid.org/0000-0003-1289-0111

Lauryn Nsenga http://orcid.org/0000-0001-6155-4718

Joseph Baruch Baluku http://orcid.org/0000-0002-5852-9674

\section{REFERENCES}

1 Marks M, Sammut T, Cabral MG, et al. The prevalence of scabies, pyoderma and other communicable dermatoses in the Bijagos Archipelago, Guinea-Bissau. PLoS Negl Trop Dis 2019;13:e0007820.

2 Amiri M, Furia FF, Bakari M. Skin disorders among children living in orphanage centres in Dar ES Salaam, Tanzania. Trop Med Health 2020;48:29.

3 Nweze El, Eke IE. Dermatophytes and dermatophytosis in the eastern and southern parts of Africa. Med Mycol 2018;56:13-28.

4 Nweze El, Eke I. Dermatophytosis in northern Africa. Mycoses 2016;59:137-44.

5 Hay RJ, Johns NE, Williams HC, et al. The global burden of skin disease in 2010: an analysis of the prevalence and impact of skin conditions. J Invest Dermatol 2014;134:1-8.

6 de Hoog GS, Dukik K, Monod M, et al. Toward a novel multilocus phylogenetic taxonomy for the dermatophytes. Mycopathologia 2017;182:5-31.

7 Zhan P, Li D, Wang C, et al. Epidemiological changes in tinea capitis over the sixty years of economic growth in China. Med Mycol 2015;53:691-8.

8 Hay RJ. Tinea capitis: current status. Mycopathologia 2017;182:87-93.

9 Rebollo N, López-Barcenas AP, Arenas R. Tinea capitis. Actas Dermo-Sifiliográficas 2008;99:91-100.

10 Farooqi M, Tabassum S, Rizvi DA, et al. Clinical types of tinea capitis and species identification in children: an experience from tertiary care centres of Karachi, Pakistan. J Pak Med Assoc 2014;64:304-8.

11 Moriarty B, Hay R, Morris-Jones R. The diagnosis and management of tinea. BMJ 2012;345:e4380.

12 Coulibaly O, L'Ollivier C, Piarroux R, et al. Epidemiology of human dermatophytoses in Africa. Med Mycol 2018;56:145-61.

13 Stroup DF, Berlin JA, Morton SC, et al. Meta-analysis of observational studies in epidemiology: a proposal for reporting. meta-analysis of observational studies in epidemiology (moose) group. JAMA 2000;283:2008-12.

14 Shamseer L, Moher D, Clarke M, et al. Preferred reporting items for systematic review and meta-analysis protocols (PRISMA-P) 2015: elaboration and explanation. BMJ 2015;349:97647. 